\title{
ACIDIFYING AND NON-ACIDIFYING CARBOXYLIC RESIN MIX- TURES USED ALONE AND WITH ACTH OR CORTISONE
}

\author{
By J. H. PETERS, T. S. DANOWSKI, L. GREENMAN, F. A. WEIGAND, C. E. CLARKE, \\ K. GARVER, F. M. MATEER, AND R. TARAIL \\ (From the Department of Research Medicine, the Renziehausen Foundation, and the Children's \\ and Presbyterian Hospitals of the University of Pittsburgh School of Medicine, \\ Pittsburgh, $P a$.)
}

(Submitted for publication April 23, 1951; accepted July 9, 1951)

Evidence has been presented indicating that a sufficient intake of a carboxylic cation exchanger in the potassium cycle increases the stool output of sodium in absolute as well as in relative terms (1-4). Two other findings accompanying the use of this form of the resin are noteworthy:a) a significant proportion of the potassium taken with or as part of the resin can be retained in the body and $b$ ) ingestion of this particular resin does not produce acidosis. Its efficiency in removing sodium is probably not as great, however, as that of either the hydrogen or the ammonium forms of the resin, each of which interferes with potassium absorption and induces a metabolic acidosis. The first half of this paper deals with findings observed during the simultaneous administration of the two types of resin in equal proportions, i.e., potassium cycle mixed with either the hydrogen or the ammonium cycle, as well as in mixtures in which the acidifying resin predominated.

The second half of this report describes results obtained during the use of resin mixtures with ACTH or cortisone or both. The expanding recourse to adrenal cortical effects in the therapy of a wide variety of disease states (5-7) has further emphasized that prolonged therapy is frequently not possible because of two particularly undesirable concomitants, i.e., sodium retention and potassium depletion. It seems probable that these side effects by necessitating interruption or intermittency of treatment deprive patients of benefits which might be otherwise obtainable. The combined resin and steroid or hormone studies herein presented describe attempts at prevention or deferment of such deviations in cation metabolism.

\section{MATERIALS AND METHODS}

Equal parts of the acidifying and non-acidifying exchange resins were administered in capsules for a daily total of 24 to $60 \mathrm{~g}$. to hospitalized children or adults during 16 periods three to seven days in length. Mixtures preponderating in the acidifying resin, i.e., 75 to $80 \%$ in the ammonium or hydrogen cycle, were given in eight subsequent studies in four patients. In the final group of experiments four parts of ammonium or hydrogen form resin and one part of the potassium resin were administered to patients undergoing treatment with adrenocorticotrophic hormone or cortisone. In all three groups of studies control periods were included when possible. The experimental procedures and other pertinent information have been described earlier $(1,2)$. The dietary regimens employed, the results of blood and serum analyses, and the available balance data are presented in tabular form. To assist interpretation the findings have been expressed statistically wherever possible and presented in graphic form.

\section{RESULTS}

\section{A. Relative constancy of serum and blood values during administration of resins in mixtures of equal proportion}

Concentrations of the various serum constituents recorded in Table $I$ at the end of the administration of equal amounts of the hydrogen and potassium cycle resins have been compared, irrespective of the dietary regimen, with the levels present prior to this treatment and expressed as increments or decrements, prefaced by the appropriate signs. The averages of these changes are depicted in Figure 1. It is readily evident that only slight fluctuations occurred in serum bicarbonate, chloride, sodium, potassium, calcium, phosphorus, protein, and water. Statistical comparison of the changes recorded during hydrogen-potassium resin ingestion, by means of the " $t$ " test with a larger group of control periods described in a preceding paper, indicates that they are indistinguishable in magnitude and direction. It is clear, therefore, that in these subjects there is no evidence of the hyperchloremia and metabolic acidosis which characteristically occurred during hydrogen cycle resin therapy of equal duration (1). It should be em- 
TABLE I

Body weight and analyses of blood and serum in subjects receiving equal parts of the $H$ and the $K$ cycle resin

\begin{tabular}{|c|c|c|c|c|c|c|c|c|c|c|c|c|c|c|}
\hline Subjece & TIme & & pr & Body Tet. & BlC & ood & & & & Serum & & & & \\
\hline (Age-Sex) & (Days) & ${ }_{8}^{(8}$ & & (kg.) & \begin{tabular}{|c|} 
Sugar \\
(mgmx)
\end{tabular} & $\underset{(m P A)}{N P A}$ & $\begin{array}{c}\mathrm{HCO}_{1} \\
(\operatorname{meq} / \mathrm{L})\end{array}$ & $\begin{array}{c}C l \\
(\text { meg./1.) }\end{array}$ & 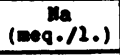 & $\begin{array}{c}K \\
\left(m \in q^{\prime} / / 2 .\right) \\
\end{array}$ & Ca & $\underset{(\operatorname{Pan} x)}{P}$ & $\begin{array}{l}H_{2}, 0 \\
. / 2.0\end{array}$ & $\begin{array}{c}\text { Protels } \\
(c .5)\end{array}$ \\
\hline $\begin{array}{l}\text { DL } \\
\text { It } F \\
\text { DIabetes }\end{array}$ & $\begin{array}{r}0 \\
0-3 \\
3-6 \\
6-9 \\
9-11\end{array}$ & $\begin{array}{l}0 \\
16 \\
16 \\
20 \\
30\end{array}$ & $\begin{array}{l}0 \\
16 \\
16 \\
20 \\
30\end{array}$ & $\begin{array}{l}50.6 \\
49.8 \\
50.4 \\
51.2 \\
51.0 \\
\end{array}$ & $\begin{array}{l}350 \\
- \\
136 \\
229 \\
- \\
\end{array}$ & $\begin{array}{l}36 \\
38 \\
34 \\
34 \\
35\end{array}$ & $\begin{array}{l}23.1 \\
21.2 \\
27.2 \\
23.9 \\
26.3 \\
\end{array}$ & $\begin{array}{r}98.8 \\
95.0 \\
100.0 \\
100.5 \\
98.3 \\
\end{array}$ & $\begin{array}{l}143 \\
142 \\
144 \\
143 \\
147\end{array}$ & & $\begin{array}{c}10.2 \\
0.8 \\
9.8 \\
9.9 \\
10.4 \\
\end{array}$ & $\begin{array}{l}.7 \\
.6 \\
.8 \\
.6 \\
\end{array}$ & $\begin{array}{l}- \\
909 \\
923 \\
925 \\
-\end{array}$ & $\begin{array}{l}8.1 \\
7.5 \\
6.6 \\
7.4 \\
8.2 \\
\end{array}$ \\
\hline $\begin{array}{l}J \\
29 \\
\text { DLabetes }\end{array}$ & $\begin{array}{r}0 \\
0-6 \\
6-11 \\
\end{array}$ & $\begin{array}{r}0 \\
20 \\
0 \\
\end{array}$ & $\begin{array}{r}0 \\
20 \\
0 \\
\end{array}$ & $\begin{array}{l}68.9 \\
68.5 \\
69.5 \\
\end{array}$ & $\begin{array}{l}69 \\
45 \\
98 \\
\end{array}$ & $\begin{array}{l}32 \\
39 \\
41 \\
\end{array}$ & $\begin{array}{l}28.1 \\
28.3 \\
26.9 \\
\end{array}$ & $\begin{array}{r}101.0 \\
101.2 \\
93.6\end{array}$ & $\begin{array}{l}147 \\
146 \\
145\end{array}$ & $\begin{array}{l}3.1 \\
5.3 \\
\end{array}$ & $\begin{array}{r}9.5 \\
10.1 \\
9.9 \\
\end{array}$ & $\begin{array}{r}4.0 \\
3.0 \\
\end{array}$ & $\begin{array}{l}931 \\
921 \\
925\end{array}$ & $\begin{array}{c}7.3 \\
7.3\end{array}$ \\
\hline 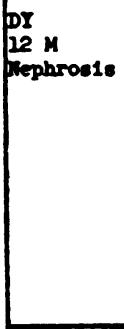 & $\begin{array}{r}0 \\
0-6 \\
10 \\
10-16 \\
16-22 \\
22-28 \\
28-35 \\
42 \\
12-46 \\
46-52 \\
68 \\
68-73 \\
73-80\end{array}$ & $\begin{array}{r}17 \\
17 \\
0 \\
0 \\
20 \\
12 \\
20 \\
\\
0 \\
33 \\
0 \\
30 \\
21\end{array}$ & $\begin{array}{r}17 \\
17 \\
0 \\
0 \\
20 \\
12 \\
20 \\
\\
0 \\
33 \\
0 \\
30 \\
21\end{array}$ & $\begin{array}{c}30.2 \\
30.4 \\
31.4 \\
31.4 \\
- \\
- \\
31.4 \\
30.8 \\
28.4 \\
-. \\
30.6 \\
31.8 \\
32.0\end{array}$ & $\begin{array}{l}67 \\
- \\
- \\
- \\
91 \\
- \\
89 \\
- \\
-\end{array}$ & $\begin{array}{l}34 \\
44 \\
33 \\
36 \\
31 \\
42 \\
40 \\
48 \\
47 \\
45 \\
38 \\
41 \\
46 \\
\end{array}$ & $\begin{array}{l}20.4 \\
23.1 \\
19.0 \\
20.5 \\
20.7 \\
19.2 \\
20.6 \\
16.2 \\
19.8 \\
17.3 \\
23.1 \\
23.7 \\
21.6 \\
\end{array}$ & $\begin{array}{l}113.6 \\
108.6 \\
113.7 \\
110.1 \\
108.4 \\
109.7 \\
109.7 \\
106.7 \\
104.1 \\
108.0 \\
101.7 \\
111.2 \\
111.1 \\
\end{array}$ & $\begin{array}{l}148 \\
141 \\
140 \\
139 \\
137 \\
145 \\
144 \\
143 \\
145 \\
135 \\
142 \\
219 \\
\end{array}$ & 3 & $\begin{array}{c}7.9 \\
6.7 \\
0 \\
7.3 \\
7.5 \\
7.3 \\
7.4 \\
6.4 \\
7.2 \\
7.8 \\
7.6 \\
7.3 \\
7.5\end{array}$ & & $\begin{array}{l}- \\
936 \\
938 \\
933 \\
931 \\
927 \\
930 \\
943 \\
926 \\
921 \\
929 \\
929 \\
-\end{array}$ & $\begin{array}{l}3.7 \\
3.5 \\
.6 \\
.12 \\
.0 \\
.8\end{array}$ \\
\hline$\sum_{5}^{x} x$ & $\begin{array}{r}0 \\
0-7 \\
7-14 \\
14-21 \\
37 \\
37-44\end{array}$ & $\begin{array}{r}0 \\
20 \\
20 \\
20 \\
0 \\
25\end{array}$ & $\begin{array}{r}0 \\
20 \\
20 \\
20 \\
0 \\
25\end{array}$ & $\begin{array}{l}17.9 \\
21.4 \\
21.5 \\
20.8 \\
20.8 \\
20.2\end{array}$ & $\begin{array}{l}76 \\
- \\
- \\
87 \\
-\end{array}$ & $\begin{array}{l}53 \\
33 \\
29 \\
28 \\
31 \\
32\end{array}$ & $\begin{array}{l}25.3 \\
21.9 \\
22.8 \\
24.1 \\
20.7 \\
23.6 \\
\end{array}$ & $\begin{array}{l}102.1 \\
102.7 \\
100.4 \\
102.3 \\
102.7 \\
100.0\end{array}$ & $\begin{array}{l}140 \\
142 \\
143 \\
139 \\
134 \\
135\end{array}$ & 5.2 & $\begin{array}{c}8.3 \\
8.2 \\
8.0 \\
8.6 \\
.\end{array}$ & $\begin{array}{l}4.6 \\
4.8 \\
5.0 \\
5.3 \\
5.0 \\
6.2 \\
\end{array}$ & $\begin{array}{l}933 \\
935 \\
932 \\
0 \\
935 \\
-\end{array}$ & $\begin{array}{l}4.4 \\
4.0 \\
3.9 \\
3.9 \\
3.9 \\
3.9\end{array}$ \\
\hline
\end{tabular}

* End of $\mathrm{H}$ resin

$\begin{array}{llllll}\mathrm{HCQ}_{3} & \mathrm{Cl} & \mathrm{Na} & \mathrm{K} & \mathrm{Ca} & \mathrm{P}\end{array}$
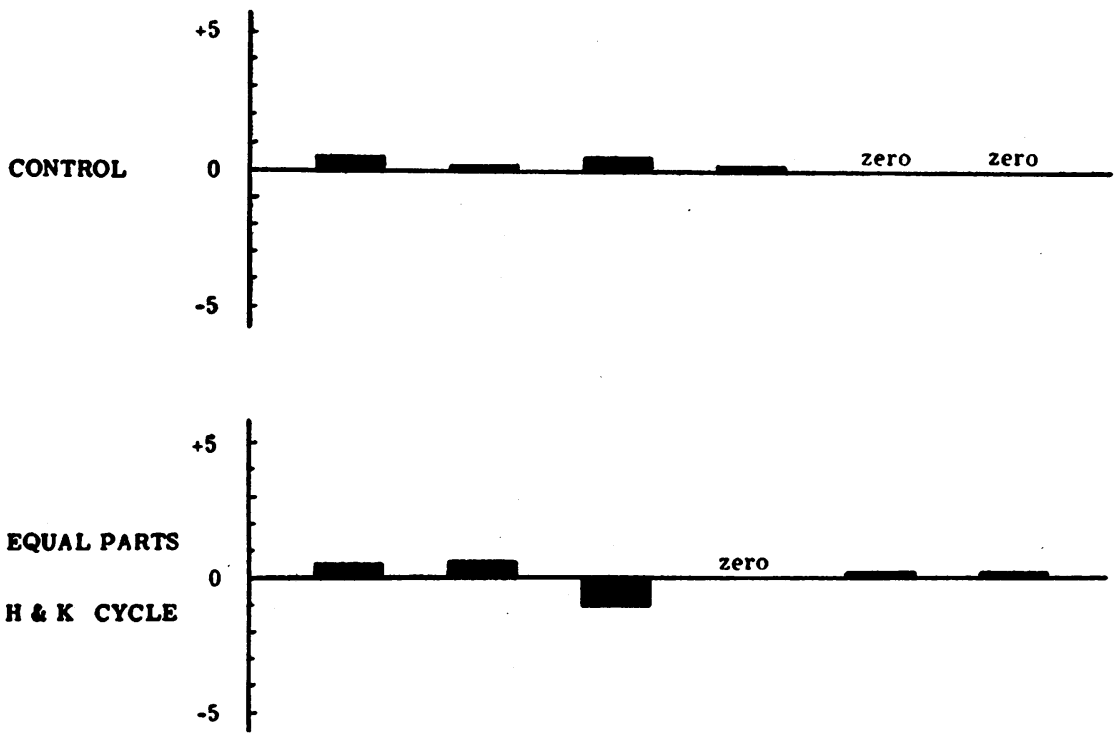

Fig. 1. Fluctuations in Serum Constituents

The columns represent mean changes in serum $\mathrm{HCO}_{8}, \mathrm{Cl}, \mathrm{Na}$, and $\mathrm{K}$ in milliequivalents per liter and in serum $\mathrm{Ca}$ and inorganic $\mathrm{P}$ in milligrams $\%$ between the onset and end of control periods and of periods in which equal parts of $H$ and $K$ cycle resins were ingested in amounts indicated in Table $\mathrm{I}$. There were no significant changes from the control values during ingestion of the resin mixture. 

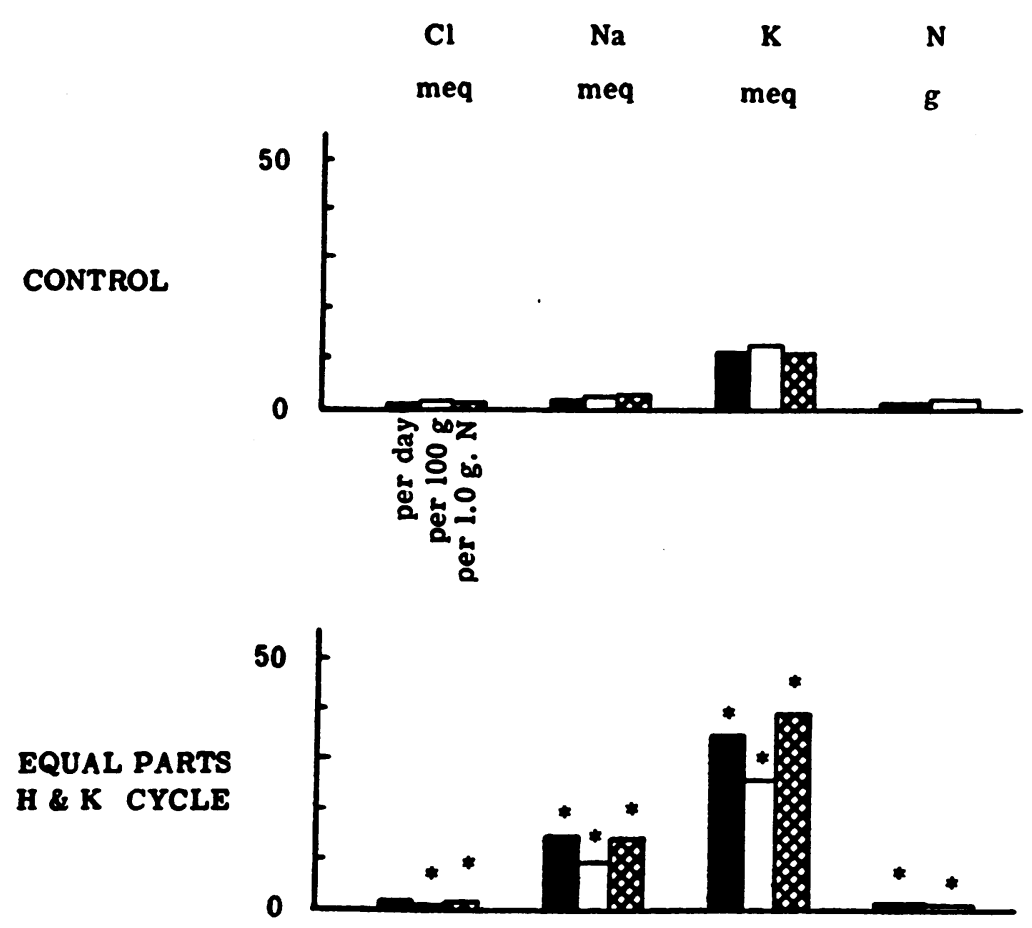

Fig. 2. Stool Data

The columns indicate the mean excretion of $\mathrm{Cl}, \mathrm{Na}, \mathrm{K}$, and $\mathrm{N}$ per day, per $100 \mathrm{~g}$. of wet stool, and per $1.0 \mathrm{~g}$. of stool nitrogen during control and resin mixture regimens. The asterisks signify statistically significant increases (" $p$ " for the " $t$ " test equals 0.05 or less) in stool excretion of all substances represented during ingestion of the resin mixture. The changes in chloride and of nitrogen are small, however.

phasized, however, that in these experiments the average intake of the hydrogen cycle cation exchanger was approximately one-half of that in the studies cited above.

Similarly, though the data are limited in number, it can be seen by inspection that the resin mixtures did not produce any definite trend in the blood sugar and nonprotein nitrogen values, and that using the ammonium rather than the hydrogen cycle resin in the mixture with the potassium cycle exchanger does not detectably alter the relative constancy of the serum or blood constituents under scrutiny (Tables I and II).

B. Acidosis and hyperchloremia observed in patients given 80: 20 mixtures of acidifying and non-acidifying resins

All of the patients receiving the exchangers in the above proportions developed some measure of acidosis. This was usually associated with a pro- portionate increase in the serum chloride. Serum sodium, potassium, calcium, phosphorus, and protein fluctuated about the pretreatment values. If the latter were within the normal range initially, and this was true in all but patient JL, the changes during therapy were not of sufficient magnitude to reach levels which were abnormally high or low (Table II).

\section{Serum and blood constituents in patients given resin mixtures in the course of $A C T H$ or cortisone therapy}

From Table III it is evident that during the simultaneous administration of ACTH or cortisone and resin mixtures the serum concentrations of bicarbonate and of chloride still decreased and increased, respectively. The findings in this limited number of observations suggest that the magnitude of the changes was somewhat less than those described earlier with the resin mixtures alone. All 
TABLE II

Body weight and analyses of blood and serum in subjects receiving $\mathrm{NH}_{4}$ and $\mathrm{K}$ cycle resins

\begin{tabular}{|c|c|c|c|c|c|c|c|c|c|c|c|c|c|c|}
\hline Subject & IIme & The & apy & Body Wet. & $B 1 C$ & ood & & & & Serum & & & & \\
\hline (Ace-Sex) & (days) & ${ }^{10}{ }^{4}$ & )$^{x}$ & (ke.) & $\begin{array}{c}\text { Sugar } \\
(\mathrm{mg} \times)\end{array}$ & $\begin{array}{c}M P 4 \\
(n / m)\end{array}$ & $\begin{array}{c}\mathrm{HCO}_{2} \\
\left(\mathrm{meq} \cdot \mathrm{N}_{.}\right) \\
\end{array}$ & $\begin{array}{c}C l \\
\left(q_{\bullet} / d_{0}\right)\end{array}$ & $(20,12)$. & 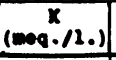 & (a) & $\left(\begin{array}{l}P \\
(m, 3)\end{array}\right.$ & $\begin{array}{l}14,00 \\
3.120)\end{array}$ & $\begin{array}{l}\text { Protele } \\
\text { (B.S) }\end{array}$ \\
\hline $\begin{array}{l}\text { RT } \\
5 \text { F } \\
\text { D1 buetes }\end{array}$ & $\begin{array}{r}0 \\
0-6\end{array}$ & $\begin{array}{l}0 \\
45\end{array}$ & $\begin{array}{r}0 \\
15\end{array}$ & $\begin{array}{l}45.0 \\
43.8\end{array}$ & 257 & $\begin{array}{l}28 \\
38\end{array}$ & $\begin{array}{l}27.2 \\
22.6\end{array}$ & $\begin{array}{l}92.6 \\
97.3\end{array}$ & $\begin{array}{l}137 \\
136\end{array}$ & $\begin{array}{l}4.8 \\
4.1\end{array}$ & 10.6 & 5.7 & $\ddot{s e 8}$ & $\ddot{7.6}$ \\
\hline $\begin{array}{l}\text { RS } \\
\text { II M } \\
\text { Diebetes }\end{array}$ & $\begin{array}{r}0 \\
0-5\end{array}$ & $\begin{array}{l}0 \\
48\end{array}$ & $\begin{array}{r}0 \\
12\end{array}$ & $\begin{array}{l}38.0 \\
37.2\end{array}$ & $\begin{array}{r}66 \\
125\end{array}$ & $\begin{array}{l}35 \\
28\end{array}$ & $\begin{array}{l}25.9 \\
18.1\end{array}$ & $\begin{array}{l}100.1 \\
104.2\end{array}$ & $\begin{array}{l}139 \\
140\end{array}$ & $\begin{array}{l}4.0 \\
4.5\end{array}$ & $\begin{array}{l}10.6 \\
10.3\end{array}$ & $\begin{array}{l}6.0 \\
6.0\end{array}$ & $\begin{array}{l}\text { 910 } \\
932\end{array}$ & $\begin{array}{l}6.1 \\
7.1\end{array}$ \\
\hline $\begin{array}{l}P C \\
13 \mathrm{M} \\
\text { Rh. fever }\end{array}$ & $\begin{array}{r}0 \\
0-7 \\
7-14 \\
14-21 \\
\end{array}$ & $\begin{array}{l}0 \\
48 \\
48 \\
48 \\
\end{array}$ & $\begin{array}{r}0 \\
12 \\
12 \\
12 \\
\end{array}$ & $\begin{array}{l}47.2 \\
44.3 \\
48.0 \\
49.1 \\
\end{array}$ & $\begin{array}{r}100 \\
79 \\
78 \\
85 \\
\end{array}$ & $\begin{array}{l}24 \\
29 \\
23 \\
21 \\
\end{array}$ & $\begin{array}{l}23.9 \\
18.3 \\
25.1 \\
23.5 \\
\end{array}$ & $\begin{array}{r}91.0 \\
100.2 \\
100.5 \\
102.8 \\
\end{array}$ & $\begin{array}{l}141 \\
139 \\
145 \\
140 \\
\end{array}$ & $\begin{array}{l}4.5 \\
4.1 \\
4.0 \\
4.0 \\
\end{array}$ & :- & $\begin{array}{l}5.5 \\
6.0 \\
5.1 \\
5.3 \\
\end{array}$ & $\begin{array}{c}\operatorname{set} \\
\text { get } \\
\text { ses } \\
-\end{array}$ & $\begin{array}{c}7.8 \\
0 \\
0.0 \\
-\end{array}$ \\
\hline $\begin{array}{l}\pi \\
14 \mathrm{M} \\
\text { GL.neph. } \\
\text { Dlabetes }\end{array}$ & $\begin{array}{r}0 \\
0-3 \\
0 \\
0-5 \\
5-14 \\
14-21 \\
\end{array}$ & $\begin{array}{l}0 \\
48 \\
0 \\
0 \\
48 \\
48\end{array}$ & $\begin{array}{r}0 \\
12 \\
0 \\
0 \\
12 \\
12 \\
\end{array}$ & $\begin{array}{c}37.0 \\
36.0 \\
37.2 \\
- \\
37.0 \\
36.0\end{array}$ & $\begin{array}{l}359 \\
153 \\
339 \\
265 \\
352 \\
348 \\
\end{array}$ & $\begin{array}{l}49 \\
66 \\
60 \\
41 \\
42 \\
48 \\
\end{array}$ & $\begin{array}{l}21.0 \\
14.2 \\
22.9 \\
27.7 \\
17.4 \\
14.2 \\
\end{array}$ & $\begin{array}{r}97.0 \\
104.0 \\
97.1 \\
90.9 \\
99.1 \\
107.6 \\
\end{array}$ & $\begin{array}{l}132 \\
148 \\
137 \\
136 \\
134 \\
132 \\
\end{array}$ & $\begin{array}{l}5.8 \\
5.6 \\
5.8 \\
5.9 \\
4.7 \\
4.5\end{array}$ & $\begin{array}{c}10.3 \\
- \\
- \\
11.8 \\
10.5 \\
9.7\end{array}$ & $\begin{array}{r}5.1 \\
5.4 \\
4.2 \\
\end{array}$ & $\begin{array}{c}930 \\
930 \\
931 \\
925 \\
929 \\
-\end{array}$ & $\begin{array}{c}7.4 \\
- \\
7.2 \\
8.1 \\
7.5 \\
- \\
\end{array}$ \\
\hline
\end{tabular}

* Calculated as $\mathrm{W}_{\mathrm{s}}=0.985-\left(0.00745 \times \mathrm{P}_{\mathrm{s}}\right)$

subjects developed hypokaliemia ranging between given. As in the earlier studies, however, the 3.4 and 3.1 meq./1. during this therapy in contrast other components of serum which were measured to the findings when ACTH or cortisone was not remained relatively constant.

TABLE III

Body weight and analyses of blood and serum in subjects receiving $\mathrm{NH}_{4}$ and $\mathrm{K}$ cycle resins together with $\mathrm{ACTH}$ or cortisone

\begin{tabular}{|c|c|c|c|c|c|c|c|c|c|c|c|c|c|c|c|}
\hline \multirow{4}{*}{$\begin{array}{l}\begin{array}{c}\text { Subject } \\
(A g e-S e x)\end{array} \\
\text { BS } \\
37 \text { F } \\
\begin{array}{c}\text { Sclero- } \\
\text { derma }\end{array}\end{array}$} & \multirow{4}{*}{$\begin{array}{l}\text { Time } \\
(\text { Days })\end{array}$} & \multicolumn{3}{|c|}{ Therapy } & \multirow{3}{*}{\begin{tabular}{|l|} 
Body \\
Wgt. \\
$(k g)$. \\
\end{tabular}} & \multirow{2}{*}{\multicolumn{2}{|c|}{ Blood }} & \multirow{2}{*}{\multicolumn{8}{|c|}{ Serum }} \\
\hline & & \multicolumn{2}{|c|}{ Resin } & \multirow{3}{*}{\begin{tabular}{|c} 
ACTH \\
$\begin{array}{c}(m g m . \\
/ d .)\end{array}$ \\
0 \\
300 \\
300
\end{tabular}} & & & & & & & & & & & \\
\hline & & $\mid \begin{array}{c}\mathrm{NH}_{(\mathrm{g}} \mid \\
\mid\end{array}$ & & & & \multirow{2}{*}{$\begin{array}{c}\begin{array}{c}\text { Sugar } \\
\text { (mgm. \%) }\end{array} \\
82 \\
47 \\
114\end{array}$} & \multirow{2}{*}{$\begin{array}{c}\begin{array}{c}\text { NPN } \\
\text { (mgm. \%) }\end{array} \\
30 \\
30 \\
43\end{array}$} & \multirow{2}{*}{\begin{tabular}{|c|c|}
$\begin{array}{c}\mathrm{HCO} \\
(\text { meq./l. })\end{array}$ \\
26.7 \\
17.3 \\
26.1
\end{tabular}} & \multirow{2}{*}{$\begin{array}{c}\mathrm{Cl} \\
(\text { meq./l. }) \\
99.5 \\
100.5 \\
99.4\end{array}$} & \multirow{2}{*}{\begin{tabular}{|c|}
$\begin{array}{c}\mathrm{Na} \\
(\text { meq./l. })\end{array}$ \\
141 \\
139 \\
135
\end{tabular}} & \multirow{2}{*}{ 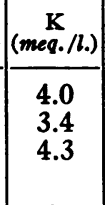 } & \multirow{2}{*}{$\begin{array}{c}\begin{array}{c}\mathrm{Ca} \\
(m g m . \%)\end{array} \\
10.4 \\
10.1 \\
9.5\end{array}$} & \multirow{2}{*}{$\begin{array}{c}\begin{array}{c}P \\
\text { (mgm. \%) }\end{array} \\
4.0 \\
4.4 \\
4.0\end{array}$} & \multirow{2}{*}{ 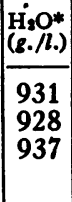 } & \multirow{2}{*}{\begin{tabular}{|c}
$\begin{array}{c}\text { Protein } \\
(\mathrm{g} . \%)\end{array}$ \\
7.2 \\
7.7 \\
6.4
\end{tabular}} \\
\hline & & $\begin{array}{r}0 \\
48 \\
0\end{array}$ & $\begin{array}{r}0 \\
12 \\
0\end{array}$ & & \begin{tabular}{|l|}
52.7 \\
50.0 \\
49.8
\end{tabular} & & & & & & & & & & \\
\hline $\begin{array}{l}\text { BM } \\
14 \text { F } \\
\text { Rh. fever }\end{array}$ & $\left|\begin{array}{c}0 \\
0-7 \\
7-14 \\
14-21\end{array}\right|$ & $\begin{array}{r}0 \\
48 \\
48 \\
48\end{array}$ & $\begin{array}{r}0 \\
12 \\
12 \\
12\end{array}$ & $\begin{array}{r}0 \\
100 \\
100 \\
100\end{array}$ & \begin{tabular}{|l|}
41.3 \\
41.1 \\
44.0 \\
45.9
\end{tabular} & $\begin{array}{l}80 \\
79 \\
98 \\
91\end{array}$ & $\begin{array}{l}37 \\
34 \\
30 \\
32\end{array}$ & $\begin{array}{l}25.5 \\
23.1 \\
24.7 \\
21.1\end{array}$ & $\begin{array}{r}97.5 \\
101.1 \\
103.1 \\
103.1\end{array}$ & $\begin{array}{l}138 \\
142 \\
137 \\
140\end{array}$ & $\begin{array}{l}4.5 \\
3.4 \\
4.0 \\
3.8\end{array}$ & E & $\begin{array}{l}5.2 \\
4.7 \\
5.0 \\
5.1\end{array}$ & $\begin{array}{l}920 \\
933 \\
-\end{array}$ & $\begin{array}{l}8.7 \\
7.0 \\
-\end{array}$ \\
\hline $\begin{array}{l}\text { BC } \\
12 \mathrm{M} \\
\text { Nephrosis }\end{array}$ & $\begin{array}{l}0 \\
0-7\end{array}$ & $\begin{array}{r}0 \\
48\end{array}$ & $\begin{array}{l}0 \\
0 \dagger\end{array}$ & $\begin{array}{r}0 \\
100\end{array}$ & \begin{tabular}{|l|}
40.2 \\
37.8
\end{tabular} & $\begin{array}{r}80 \\
100\end{array}$ & $\begin{array}{l}39 \\
69\end{array}$ & $\begin{array}{l}21.8 \\
11.8\end{array}$ & $\begin{array}{l}109.4 \\
120.8\end{array}$ & $\begin{array}{l}136 \\
138\end{array}$ & $\begin{array}{l}3.6 \\
3.2\end{array}$ & $\begin{array}{l}6.0 \\
6.6\end{array}$ & $\begin{array}{l}5.5 \\
5.4\end{array}$ & 二 & 二 \\
\hline $\begin{array}{l}\mathrm{RL} \\
42 \mathrm{M} \\
\text { Hemolytic } \\
\text { anemia }\end{array}$ & $\begin{array}{l}0 \\
0-7 \\
7-13\end{array}$ & $\begin{array}{r}0 \\
48 \\
48\end{array}$ & $\begin{array}{r}0 \\
12 \\
12\end{array}$ & $\begin{array}{l}200 \ddagger \\
200 \ddagger \\
200 \ddagger\end{array}$ & $\begin{array}{l}57.3 \\
57.7 \\
57.7\end{array}$ & $\frac{80}{77}$ & $\begin{array}{l}36 \\
46 \\
33\end{array}$ & $\begin{array}{l}29.5 \\
29.5 \\
27.5\end{array}$ & $\begin{array}{l}99.2 \\
98.0 \\
95.3\end{array}$ & $\begin{array}{l}139 \\
144 \\
137\end{array}$ & $\begin{array}{l}4.4 \\
3.2 \\
4.1\end{array}$ & $\overline{\overline{9.5}}$ & $\overline{\overline{4.6}}$ & $\begin{array}{l}937 \\
937 \\
932\end{array}$ & $\begin{array}{l}6.5 \\
6.4 \\
7.1\end{array}$ \\
\hline $\begin{array}{l}\text { JM } \\
20 \mathrm{M} \\
\text { Aplastic } \\
\text { anemia }\end{array}$ & $\begin{array}{l}0 \\
0-7\end{array}$ & $\begin{array}{c}0 \\
488\end{array}$ & $\begin{array}{r}0 \\
12\end{array}$ & $\begin{array}{l}300 \\
300\end{array}$ & 80.0 & $\begin{array}{l}75 \\
97\end{array}$ & $\begin{array}{l}32 \\
36\end{array}$ & $\begin{array}{l}30.2 \\
25.2\end{array}$ & $\begin{array}{l}99.8 \\
97.2\end{array}$ & $\begin{array}{l}140 \\
143\end{array}$ & $\begin{array}{l}3.8 \\
3.1\end{array}$ & $\stackrel{8.4}{-}$ & $\begin{array}{l}4.0 \\
4.2\end{array}$ & 945 & 5.4 \\
\hline
\end{tabular}

* Calculated as $W_{0}=0.985-(0.00745)\left(P_{8}\right)$

$\dagger$ Ingested 50 meq. KHCO $/$ day instead of $\mathrm{K}$ cycle resin

$\ddagger$ Oral cortisone instead of ACTH

$\$ \mathrm{H}$ cycle resin administered instead of $\mathrm{NH}_{4}$ cycle 
TABLE IV

Intake data and urine and stool output in subjects receiving equal parts of the $H$ and the $K$ cycle resin

\begin{tabular}{|c|c|c|c|c|c|c|c|c|c|c|c|c|c|c|c|c|c|c|c|}
\hline \multirow{2}{*}{ Subj. } & \multirow{2}{*}{$\begin{array}{c}\text { Time } \\
\text { (Days) }\end{array}$} & \multicolumn{2}{|c|}{ Therapy } & \multicolumn{5}{|c|}{ Intake } & \multicolumn{6}{|c|}{ Urine } & \multicolumn{5}{|c|}{ Stool } \\
\hline & & \multicolumn{2}{|c|}{$\underset{(g . / d .)}{\mathbf{H}} \mid \mathbf{K}$} & $\underset{(l .)}{\text { Fluid }}$ & $\underset{\text { (meq.) }}{\mathrm{Cl}}$ & $\underset{(\text { meq. })}{\mathrm{Na}}$ & $\underset{(\text { meq. })}{\mathbf{K}}$ & $\underset{(g .)}{\mathbf{N}}$ & $\begin{array}{l}\text { Vol. } \\
(l .)\end{array}$ & $\underset{\text { (meq.) }}{\mathrm{Cl}}$ & $\underset{\text { (meq.) }}{\mathrm{Na}}$ & $\underset{\text { (meq. })}{\mathbf{K}}$ & TN & $\underset{(g .)}{\text { NPN }}$ & $\underset{(\mathrm{g} .)}{\text { Wgt. }}$ & $\underset{\text { (meq. })}{\mathrm{Cl}}$ & $\underset{\text { (meq.) }}{\mathrm{Na}}$ & $\underset{\text { (meq.) }}{\mathbf{K}}$ & $\underset{(g .)}{N}$ \\
\hline $\mathrm{DL}$ & $\begin{array}{l}0-6 \\
6-9 \\
9-11\end{array}$ & $\begin{array}{l}16 \\
20 \\
30\end{array}$ & $\begin{array}{l}16 \\
20 \\
30\end{array}$ & \begin{tabular}{|r|}
20.74 \\
9.99 \\
7.30
\end{tabular} & $\begin{array}{l}506 \\
250 \\
167\end{array}$ & $\begin{array}{l}424 \\
210 \\
140\end{array}$ & $\begin{array}{r}1070 \\
523 \\
424 \\
\end{array}$ & $\begin{array}{l}88.1 \\
43.5 \\
29.0\end{array}$ & $\begin{array}{l}\overline{7.30} \\
4.95\end{array}$ & $\begin{array}{l}\overline{135} \\
152\end{array}$ & $\begin{array}{r}-7 \\
52\end{array}$ & $\begin{array}{l}\overline{353} \\
260\end{array}$ & $\begin{array}{l}\overrightarrow{37.3} \\
22.8\end{array}$ & 二 & $\begin{array}{r}720 \\
66\end{array}$ & $\begin{array}{l}7 \\
1\end{array}$ & $\begin{array}{l}54 \\
14\end{array}$ & $\begin{array}{r}144 \\
50\end{array}$ & $\begin{array}{l}3.3 \\
0.7\end{array}$ \\
\hline JF & $\begin{array}{l}0-6 \\
6-11\end{array}$ & $\begin{array}{r}20 \\
0\end{array}$ & $\begin{array}{r}20 \\
0\end{array}$ & \multicolumn{5}{|c|}{ Full diab. diet } & $\begin{array}{r}11.50 \\
8.52\end{array}$ & $\begin{array}{r}1799 \\
480\end{array}$ & $\begin{array}{l}1371 \\
1258\end{array}$ & $\begin{array}{l}740 \\
349\end{array}$ & $\begin{array}{l}91.7 \\
63.2\end{array}$ & $=$ & 1073 & $\frac{2}{-}$ & 36 & 203 & 6.2 \\
\hline \multirow[t]{2}{*}{ DY } & \multirow{2}{*}{$\begin{array}{c}0-6 \\
10-16 \\
16-22 \\
22-28 \\
28-35 \\
42-46 \\
46-52 \\
68-73 \\
73-80\end{array}$} & \multirow{2}{*}{$\begin{array}{r}17 \\
0 \\
20 \\
12 \\
20 \\
0 \\
33 \\
30 \\
21\end{array}$} & \multirow{2}{*}{$\begin{array}{r}17 \\
0 \\
20 \\
12 \\
20 \\
0 \\
33 \\
30 \\
21\end{array}$} & \begin{tabular}{|r|}
11.03 \\
13.67 \\
13.67 \\
17.29 \\
8.10
\end{tabular} & $\begin{array}{c}r \\
146 \\
192 \\
180 \\
192 \\
64\end{array}$ & $\begin{array}{r}\text { ull die } \\
9 \\
11 \\
11 \\
11 \\
4\end{array}$ & \begin{tabular}{|l|}
354 \\
467 \\
438 \\
466 \\
156
\end{tabular} & $\begin{array}{l}65.0 \\
83.7 \\
80.4 \\
85.5 \\
28.6 \\
\end{array}$ & $\begin{array}{r}10.70 \\
7.05 \\
6.70 \\
9.60 \\
8.48 \\
8.10\end{array}$ & $\begin{array}{l}840 \\
338 \\
328 \\
343 \\
309 \\
427\end{array}$ & $\begin{array}{l}792 \\
197 \\
114 \\
216 \\
119 \\
383\end{array}$ & $\begin{array}{l}654 \\
270 \\
279 \\
468 \\
108 \\
198\end{array}$ & \begin{tabular}{|l}
73.2 \\
58.0 \\
55.7 \\
67.5 \\
73.9 \\
42.8
\end{tabular} & $\begin{array}{l}61.4 \\
45.3 \\
46.1 \\
54.0 \\
63.1 \\
33.8\end{array}$ & $\begin{array}{r}838 \\
1171 \\
983 \\
981 \\
758 \\
677\end{array}$ & $\begin{array}{r}2 \\
11 \\
11 \\
12 \\
7 \\
1\end{array}$ & $\begin{array}{l}60 \\
26 \\
35 \\
43 \\
33 \\
23\end{array}$ & $\begin{array}{r}193 \\
48 \\
167 \\
124 \\
149 \\
13\end{array}$ & $\begin{array}{l}8.4 \\
6.3 \\
9.6 \\
9.2 \\
9.7 \\
3.4\end{array}$ \\
\hline & & & & \multicolumn{5}{|c|}{ "Salt-free" diet } & $\begin{array}{l}14.20 \\
11.00 \\
14.10 \\
\end{array}$ & $\begin{array}{r}340 \\
0 \\
194 \\
\end{array}$ & $\begin{array}{r}213 \\
23 \\
150 \\
\end{array}$ & $\begin{array}{l}454 \\
403 \\
661\end{array}$ & $\begin{array}{l}71.1 \\
48.6 \\
84.9 \\
\end{array}$ & $\begin{array}{l}60.1 \\
38.7 \\
69.8\end{array}$ & $\begin{array}{r}981 \\
1061 \\
2007\end{array}$ & $\begin{array}{r}5 \\
13\end{array}$ & $\begin{array}{r}158 \\
93 \\
58 \\
\end{array}$ & $\begin{array}{l}241 \\
271 \\
125\end{array}$ & $\begin{array}{r}7.6 \\
9.5 \\
13.1 \\
\end{array}$ \\
\hline \multirow[t]{2}{*}{ BF } & $0-7$ & 20 & 20 & \multicolumn{5}{|c|}{ Full diet } & 5.38 & 177 & 9 & 288 & 39.4 & 36.5 & & 14 & 318 & 614 & 18.8 \\
\hline & $\begin{array}{r}7-14 \\
14-21 \\
37-44\end{array}$ & $\begin{array}{l}20 \\
20 \\
25\end{array}$ & $\begin{array}{l}20 \\
20 \\
25\end{array}$ & \multicolumn{5}{|c|}{ "Salt-free" diet } & $\begin{array}{l}6.45 \\
5.75 \\
7.00\end{array}$ & $\begin{array}{l}259 \\
170 \\
124\end{array}$ & $\begin{array}{l}58 \\
23 \\
50\end{array}$ & $\begin{array}{l}505 \\
281 \\
383\end{array}$ & \begin{tabular}{|l|}
36.6 \\
31.5 \\
40.2
\end{tabular} & $\begin{array}{l}34.2 \\
29.3 \\
35.8\end{array}$ & $\begin{array}{r}881 \\
1310\end{array}$ & $\begin{array}{r}3 \\
3 \\
4\end{array}$ & $\overline{149}$ & $\begin{array}{l}333 \\
690\end{array}$ & $\begin{array}{r}7.00 \\
7.2 \\
9.1\end{array}$ \\
\hline
\end{tabular}

D. Stool composition during ingestion of cation exchange resins in two different cycles (Table IV)

As in all of the earlier studies with the individual resins (1) there is no evidence that these several forms of the resin in combination alter the output of either chloride or of nitrogen per day, per unit of stool mass, or per unit of stool nitrogen. The second line of Figure 2 expresses mathematically the stool data in Table IV in the 14 periods in which the subjects received the resins in equal proportions while on a full or a so-called hospital "saltfree" diet. The exclusion of the three periods in which patient DY was maintained on a regimen markedly restricted as to sodium permits comparison of data derived from dietetically homogeneous groups. This is necessary because of the demonstration that maintenance on a no-salt milk formula of the type which subject DY received in itself alters stool composition (8). The control material (Figure 2, line 1) repeated from an earlier paper, represents therefore the average content of chloride, sodium, potassium, and nitrogen to be expected in the stools of individuals receiving a substantial amount of sodium, whether it be in milk or in a diet that is or is not labelled "salt-free." It is readily evident that the hydrogen-potassium resin

TABLE V

Intake data and urine and stool output in subjects receiving $\mathrm{NH}_{4}$ and $\mathrm{K}$ cycle resins

\begin{tabular}{|c|c|c|c|c|c|c|c|c|c|c|c|c|c|c|c|c|c|c|}
\hline \multirow{2}{*}{ Subj. } & \multirow{2}{*}{$\underset{(D \text { Days })}{\text { Time }}$} & \multicolumn{2}{|c|}{ Therapy } & \multicolumn{5}{|c|}{ Intake } & \multicolumn{5}{|c|}{ Urine } & \multicolumn{5}{|c|}{ Stool } \\
\hline & & \multicolumn{2}{|c|}{$\left.\underset{\left(g_{0} / d .\right)}{\mathrm{NH}_{4}}\right|^{\mathrm{K}}$} & $\begin{array}{c}\text { Fluid } \\
(l .)\end{array}$ & $\underset{(\text { meq. })}{\mathrm{Cl}}$ & $\underset{(\text { meq. }}{\mathrm{Na}}$ & $\underset{(\text { meq. })}{\mathbf{K}}$ & $\begin{array}{c}\mathbf{N} \\
(\mathbf{g} .)\end{array}$ & $\begin{array}{l}\text { Vol. } \\
\text { (l.) }\end{array}$ & $\underset{(\text { meq. })}{\mathrm{Cl}}$ & $\begin{array}{c}\mathrm{Na} \\
(\text { meq. })\end{array}$ & $\underset{(\text { meq. })}{\mathbf{K}}$ & $\begin{array}{l}\text { TN } \\
(\mathrm{g} .) \\
\end{array}$ & $\begin{array}{c}\text { Wgt. } \\
\text { (g.) }\end{array}$ & $\underset{\text { (meq. })}{\mathrm{Cl}}$ & $\underset{(\text { meq. })}{\mathrm{Na}}$ & $\underset{(\text { meq. })}{\mathbf{K}}$ & $\begin{array}{c}\mathbf{N} \\
(\boldsymbol{g} .) \\
\end{array}$ \\
\hline $\mathrm{RT}$ & $0-6$ & 45 & 15 & 21.32 & 363 & 306 & 773 & 96.4 & 13.72 & 383 & 64 & 329 & 101.5 & 1058 & 6 & 358 & 620 & 4.6 \\
\hline RS & $0-5$ & 48 & 12 & 22.04 & 434 & 364 & 743 & 114.8 & 12.28 & 304 & 95 & 230 & 79.8 & 294 & 9 & 27 & 168 & 3.0 \\
\hline \multirow[t]{2}{*}{ FC } & $0-7$ & 48 & 12 & 19.94 & 415 & 348 & 815 & 109.8 & 10.63 & 503 & 159 & 269 & 81.8 & 1471 & 14 & 99 & 520 & 9.1 \\
\hline & $\begin{array}{r}7-14 \\
14-21 \\
\end{array}$ & $\begin{array}{l}48 \\
48 \\
\end{array}$ & $\begin{array}{l}12 \\
12 \\
\end{array}$ & \multicolumn{5}{|c|}{ Full diet } & $\begin{array}{l}7.30 \\
5.95\end{array}$ & $\begin{array}{l}856 \\
703 \\
\end{array}$ & 269 & $\begin{array}{l}166 \\
209\end{array}$ & $\begin{array}{l}67.4 \\
50.3\end{array}$ & $\begin{array}{l}1006 \\
1437\end{array}$ & $\begin{array}{r}8 \\
10\end{array}$ & $\begin{array}{l}343 \\
295\end{array}$ & $\begin{array}{l}273 \\
415\end{array}$ & $\begin{array}{r}8.0 \\
10.9\end{array}$ \\
\hline \multirow[t]{2}{*}{ JL } & $0-3$ & 48 & 12 & 12.52 & 260 & 218 & 446 & 68.9 & 7.80 & 233 & 131 & 264 & 54.6 & 110 & 1 & 71 & 78 & 0.6 \\
\hline & $\begin{array}{c}0-5 \\
5-14 \\
14-21\end{array}$ & $\begin{array}{r}0 \\
48 \\
48\end{array}$ & $\begin{array}{r}0 \\
12 \\
12\end{array}$ & \multicolumn{5}{|c|}{ "Salt-free" diet } & $\begin{array}{r}1.40 \\
15.40 \\
14.50\end{array}$ & $\begin{array}{r}26 \\
145 \\
146\end{array}$ & $\begin{array}{r}18 \\
148 \\
72\end{array}$ & $\begin{array}{r}49 \\
476 \\
186\end{array}$ & $\begin{array}{r}8.0 \\
117.0 \\
101.5\end{array}$ & $\begin{array}{r}623 \\
1230 \\
1547\end{array}$ & $\begin{array}{r}2 \\
2 \\
14\end{array}$ & $\begin{array}{r}2 \\
14\end{array}$ & $\begin{array}{r}37 \\
198 \\
593\end{array}$ & $\begin{array}{r}4.4 \\
8.4 \\
12.2\end{array}$ \\
\hline
\end{tabular}


mixture taken in equal amounts raises the stool output of potassium in absolute values to a greater degree than stool sodium.

On the other hand, examination of the intake and excretion data in the children and adults given the resin mixtures which contained predominantly hydrogen or ammonium form resins (Table V), clearly indicates that the stool sodium and potassium values exceeded by a statistically significant margin the mean output of these ele-

TABLE VI

Intake data and urine and stool output in subjects receiving $\mathrm{NH}_{4}$ and $\mathrm{K}$ cycle resins together with $A C T H$ or cortisone

\begin{tabular}{|c|c|c|c|c|c|c|c|c|c|c|c|c|c|c|c|c|c|c|c|}
\hline \multirow{4}{*}{$\begin{array}{l}\text { Subj. } \\
\text { BS }\end{array}$} & \multirow{4}{*}{$\begin{array}{c}\begin{array}{c}\text { Time } \\
(\text { Days })\end{array} \\
\begin{array}{l}0-5 \\
5-11\end{array}\end{array}$} & \multicolumn{3}{|c|}{ Therapy } & \multirow{2}{*}{\multicolumn{5}{|c|}{ Intake }} & \multirow{2}{*}{\multicolumn{5}{|c|}{ Urine }} & \multirow{2}{*}{\multicolumn{5}{|c|}{ Stool }} \\
\hline & & \multicolumn{2}{|c|}{ Resin } & \multirow{2}{*}{$\frac{\mathrm{ACTH}}{\left(\begin{array}{c}\text { mgm. } \\
/ d .)\end{array}\right.}$} & & & & & & & & & & & & & & & \\
\hline & & \multicolumn{2}{|c|}{$\underset{(g . / d .)}{\mathrm{NH}_{1} \mid} \mathbf{K}$} & & $\underset{(l .)}{\text { Fluid }}$ & $\underset{(\text { meq. })}{\mathrm{Cl}}$ & $\underset{(\text { meq.) }}{\mathrm{Na}}$ & $\underset{(\text { meg. })}{\mathbf{K}}$ & $\underset{(\boldsymbol{g})}{N}$ & $\begin{array}{l}\text { Vol. } \\
(l .)\end{array}$ & $\underset{(\text { meq. })}{\mathrm{Cl}}$ & $\underset{(\text { meq. })}{\mathbf{N a}}$ & $\underset{(\text { meq. })}{\mathbf{K}}$ & $\begin{array}{l}\mathrm{TN} \\
(\mathrm{g} .)\end{array}$ & $\underset{(g .)}{\text { Wgt. }}$ & $\underset{(\text { meq. })}{\mathrm{Cl}}$ & $\underset{(\text { meq. })}{\mathrm{Na}}$ & $\underset{\text { (meq.) }}{\mathbf{K}}$ & $\underset{(g .)}{N}$ \\
\hline & & $\begin{array}{r}48 \\
0\end{array}$ & $\begin{array}{r}12 \\
0 \\
\end{array}$ & $\begin{array}{l}300 \\
300\end{array}$ & $\begin{array}{l}12.06 \\
10.73 \\
\end{array}$ & $\begin{array}{l}119 \\
113\end{array}$ & $\begin{array}{l}7 \\
7 \\
\end{array}$ & $\begin{array}{l}518 \\
272\end{array}$ & $\begin{array}{r}52.9 \\
50.0\end{array}$ & $\begin{array}{r}10.25 \\
6.20\end{array}$ & $\begin{array}{r}303 \\
90\end{array}$ & $\begin{array}{r}102 \\
26\end{array}$ & $\begin{array}{r}287 \\
68\end{array}$ & $\begin{array}{l}88.2 \\
83.1\end{array}$ & $\begin{array}{l}700 \\
338\end{array}$ & $\mathbf{3}$ & $\begin{array}{r}181 \\
57\end{array}$ & $\begin{array}{l}373 \\
235\end{array}$ & $\begin{array}{l}5.2 \\
2.3\end{array}$ \\
\hline \multirow[t]{2}{*}{$\mathbf{B M}$} & \multirow{2}{*}{$\begin{array}{c}0-7 \\
7-14 \\
14-21 \\
\end{array}$} & \multirow{2}{*}{$\begin{array}{l}48 \\
48 \\
48 \\
\end{array}$} & \multirow{2}{*}{$\begin{array}{l}12 \\
12 \\
12 \\
\end{array}$} & \multirow{2}{*}{$\begin{array}{l}100 \\
100 \\
100 \\
\end{array}$} & 16.54 & 411 & 345 & 806 & 108.7 & \multirow{2}{*}{\begin{tabular}{|r|}
10.36 \\
6.20 \\
8.93 \\
\end{tabular}} & \multirow{2}{*}{$\begin{array}{r}383 \\
872 \\
1100\end{array}$} & \multirow{2}{*}{$\begin{array}{l}210 \\
685 \\
893\end{array}$} & \multirow{2}{*}{$\begin{array}{l}264 \\
222 \\
232\end{array}$} & \multirow{2}{*}{$\begin{array}{l}87.0 \\
77.1 \\
80.4\end{array}$} & \multirow{2}{*}{$\begin{array}{l}1592 \\
1577\end{array}$} & \multirow{2}{*}{$\begin{array}{l}34 \\
17\end{array}$} & \multirow{2}{*}{$\begin{array}{l}259 \\
251\end{array}$} & \multirow{2}{*}{$\begin{array}{l}504 \\
512\end{array}$} & \multirow{2}{*}{$\begin{array}{l}12.2 \\
12.3\end{array}$} \\
\hline & & & & & \multicolumn{5}{|c|}{ Full diet } & & & & & & & & & & \\
\hline $\overrightarrow{\mathrm{BC}}$ & $0-7$ & 48 & $0^{*}$ & 100 & \multicolumn{5}{|c|}{ "Salt-free" diet } & - & - & - & - & - & $\overline{853}$ & 2 & 374 & 358 & 6.7 \\
\hline $\mathbf{R L}$ & $\begin{array}{l}0-7 \\
7-13 \\
\end{array}$ & $\begin{array}{l}48 \\
48 \\
\end{array}$ & $\begin{array}{l}12 \\
12 \\
\end{array}$ & $\begin{array}{l}200 \dagger \\
200 \dagger\end{array}$ & \multicolumn{5}{|c|}{ "Salt-free" diet } & $\overline{17.20}$ & $\overline{918}$ & $\overline{705}$ & $\overline{471}$ & $\overline{72.2}$ & $\begin{array}{l}1284 \\
2434\end{array}$ & $\begin{array}{r}6 \\
23\end{array}$ & $\begin{array}{l}101 \\
376\end{array}$ & $\begin{array}{l}556 \\
724\end{array}$ & $\begin{array}{l}13.8 \\
20.9\end{array}$ \\
\hline $\mathbf{J M}$ & $0-7$ & $48 \mp$ & 12 & 300 & 16.35 & 294 & 261 & 553 & 73.7 & 8.70 & 393 & 177 & 244 & 136.6 & 1515 & 2 & 299 & 538 & 5.3 \\
\hline
\end{tabular}

* 50 meq. $\mathrm{KHCO}_{3}$ ingested per day

+ Oral cortisone

$\ddagger$ Received $\mathrm{H}$ cycle resin instead of $\mathrm{NH}_{4}$

TABLE VII

External, extracellular, and cell balances in subjects receiving equal parts of the $H$ and the $K$ cycle resin

\begin{tabular}{|c|c|c|c|c|c|c|c|c|c|c|c|c|c|c|}
\hline \multirow[t]{2}{*}{ Subject } & \multirow{2}{*}{ Tine } & \multicolumn{2}{|c|}{ Therapy } & \multicolumn{4}{|c|}{ Ext. Bal. } & \multicolumn{3}{|c|}{ Extracell. Bal. } & \multicolumn{3}{|c|}{ Cell Bal. } & \\
\hline & & B & (d.) $)^{K}$ & $\begin{array}{c}C 2 \\
(\mathrm{meg} .)\end{array}$ & $\begin{array}{c}10 \\
(m e q .)\end{array}$ & $\begin{array}{c}K \\
(\operatorname{meq},)\end{array}$ & $(0)$ & $\begin{array}{l}\text { h,o } \\
(1 .)\end{array}$ & (ned.) & $\left(\begin{array}{c}x \\
\left.g_{0}\right)\end{array}\right.$ & $\left(g^{n} \cdot\right)$ & $(\operatorname{meq})$. & $\begin{array}{c}R \\
(\operatorname{mog} .)\end{array}$ & \\
\hline DL & $\begin{array}{r}6-9 \\
9-12\end{array}$ & $\begin{array}{l}20 \\
30\end{array}$ & $\begin{array}{l}20 \\
30\end{array}$ & $\begin{array}{l}+112 \\
+11\end{array}$ & $\begin{array}{r}+193 \\
+\quad 79\end{array}$ & $\begin{array}{r}+150 \\
+134 \\
\end{array}$ & $\begin{array}{l}+5.5 \\
+5.6 \\
\end{array}$ & $\begin{array}{l}+0.9 \\
+0.4\end{array}$ & $\begin{array}{l}+119 \\
+202 \\
\end{array}$ & $\begin{array}{l}+11 \\
-6 \\
\end{array}$ & $\begin{array}{r}5.5 \\
+5.3 \\
\end{array}$ & $\begin{array}{l}474 \\
-23\end{array}$ & $\begin{array}{r}+139 \\
+140 \\
\end{array}$ & $\begin{array}{l}1266 \\
127\end{array}$ \\
\hline DI & $\begin{array}{l}10-16 \\
16-22 \\
22-28 \\
28-35 \\
42-46\end{array}$ & $\begin{array}{r}0 \\
20 \\
12 \\
20 \\
0\end{array}$ & $\begin{array}{r}0 \\
20 \\
12 \\
20 \\
0\end{array}$ & $\begin{array}{l}-206 \\
-149 \\
-177 \\
-127 \\
-367\end{array}$ & $\begin{array}{l}-217 \\
-141 \\
-252 \\
-144 \\
-406\end{array}$ & $\begin{array}{r}+36 \\
+21 \\
-154 \\
+209 \\
-55\end{array}$ & $\begin{array}{r}+0.5 \\
+18.2 \\
+3.7 \\
+1.7 \\
-17.8\end{array}$ & $\begin{array}{l}-1.41 \\
-1.1 \\
-1.5 \\
-1.0 \\
-3.1\end{array}$ & $\begin{array}{l}-201 \\
-167 \\
-165 \\
-161 \\
-408\end{array}$ & $\begin{array}{l}+4 \\
-6 \\
-5 \\
+6 \\
-12\end{array}$ & $\begin{array}{r}-0.1 \\
+19.2 \\
+1.7 \\
+1.9 \\
-17.6\end{array}$ & $\begin{array}{l}-26 \\
126 \\
-87 \\
+17 \\
+2\end{array}$ & $\begin{array}{r}32 \\
+27 \\
-149 \\
+203 \\
-43\end{array}$ & $\begin{array}{l}13 \\
-19 \\
-153 \\
+199 \\
-\quad 1\end{array}$ \\
\hline
\end{tabular}

* Balances of $\mathrm{N}$ in excess of transfers of NPN

** Value corrected for anabolism or catabolism of protein

\# In view of persistent edema, chloride space in this patient was taken to equal $30 \%$ of body weight

TABLE VIII

External, extracellular, and cell balances in subjects receiving $N H_{4}$ and $K$ cycle resins

\begin{tabular}{|c|c|c|c|c|c|c|c|c|c|c|c|c|c|c|}
\hline \multirow{3}{*}{$\begin{array}{c}\text { Subject } \\
\text { RT }\end{array}$} & \multirow{3}{*}{$\begin{array}{c}\begin{array}{c}\text { Time } \\
(\text { Days })\end{array} \\
0-6\end{array}$} & \multicolumn{2}{|c|}{ Therapy } & \multicolumn{4}{|c|}{ External bal. } & \multicolumn{3}{|c|}{ Extracell. bal. } & \multicolumn{4}{|c|}{ Cell. bal. } \\
\hline & & \multicolumn{2}{|c|}{$\underset{(g . / d .)}{\mathrm{NH}_{4}} \mathrm{~K}$} & $\underset{(m e q .)}{\mathrm{Cl}}$ & $\underset{(\text { meq. })}{\mathrm{Na}}$ & $\underset{(m e q .)}{\mathbf{K}}$ & $\underset{(\boldsymbol{g} .)}{\mathbf{N}}$ & $\underset{(l .)}{\mathrm{H}_{2} \mathrm{O}}$ & $\underset{\text { (meq.) }}{\mathrm{Na}}$ & $\underset{\text { (meq.) }}{\mathbf{K}}$ & $\begin{array}{l}N^{*} \\
(\mathrm{~g} .)\end{array}$ & $\underset{(\text { meq. })}{\mathrm{Na}}$ & $\underset{\text { (meq.) }}{\mathbf{K}}$ & $\underset{(\text { meq. })}{\mathbf{K \dagger}}$ \\
\hline & & 45 & 15 & -27 & -120 & -176 & -10.0 & -0.7 & -100 & -8 & -12.8 & -20 & -168 & -138 \\
\hline $\mathbf{R S}$ & $0-5$ & 48 & 12 & +118 & +238 & +345 & +31.7 & +0.6 & +103 & +7 & +33.4 & +135 & +338 & +258 \\
\hline FC & $0-7$ & 48 & 12 & -105 & +86 & +26 & +18.6 & -1.2 & -189 & -8 & +17.2 & +275 & +34 & -7 \\
\hline JL & $0-3$ & 48 & 12 & +24 & +12 & +104 & +13.4 & -0.3 & +79 & -3 & +9.4 & -67 & +107 & +85 \\
\hline
\end{tabular}

* Balance of $\mathrm{N}$ in excess of transfers of NPN

$\uparrow$ Values corrected for anabolism and catabolism of protein 
TABLE IX

External, extracellular, and cell balances in subjects receiving $N_{4}$ and $K$ cycle resins together with $A C T H$ or cortisone

\begin{tabular}{|c|c|c|c|c|c|c|c|c|c|c|c|c|c|c|c|}
\hline \multirow{4}{*}{$\begin{array}{c}\text { Subject } \\
\text { BS }\end{array}$} & \multirow{4}{*}{$\begin{array}{c}\begin{array}{c}\text { Time } \\
(\text { Days })\end{array} \\
\begin{array}{l}0-5 \\
5-11\end{array}\end{array}$} & \multicolumn{3}{|c|}{ Therapy } & \multirow{2}{*}{\multicolumn{4}{|c|}{ External bal. }} & \multirow{2}{*}{\multicolumn{3}{|c|}{ Extracell. bal. }} & \multirow{2}{*}{\multicolumn{4}{|c|}{ Cell. bal. }} \\
\hline & & \multicolumn{2}{|c|}{ Resin } & \multirow{2}{*}{$\begin{array}{c}\text { АCTH } \\
\text { (mgm./d. })\end{array}$} & & & & & & & & & & & \\
\hline & & $\underset{(\mathrm{g} .}{\mathrm{NH}_{4}}$ & d.) & & $\underset{\text { (meq.) }}{\mathrm{Cl}}$ & $\underset{\text { (meq.) }}{\mathrm{Na}}$ & $\underset{\text { (meq.) }}{\mathbf{K}}$ & $\underset{(8 .)}{N}$ & $\begin{array}{c}\mathrm{H}_{2} \mathrm{O} \\
(l .)\end{array}$ & $\underset{\text { (meq.) }}{\mathrm{Na}}$ & $\underset{\text { (meq.) }}{\mathbf{K}}$ & $\begin{array}{l}N^{*} \\
(g .)\end{array}$ & $\underset{(\text { meq. })}{\mathrm{Na}}$ & $\underset{\text { (meq.) }}{\mathbf{K}}$ & $\underset{\text { (meq.) }}{\mathbf{K \dagger}}$ \\
\hline & & $\begin{array}{r}48 \\
0\end{array}$ & $\begin{array}{r}12 \\
0\end{array}$ & $\begin{array}{l}300 \\
300\end{array}$ & $\begin{array}{l}-190 \\
+\quad 18\end{array}$ & $\begin{array}{l}-280 \\
-79\end{array}$ & $\begin{array}{l}-142 \\
-31\end{array}$ & $\begin{array}{l}-40.8 \\
-35.7\end{array}$ & $\begin{array}{l}-1.8 \\
+0.3\end{array}$ & $\begin{array}{r}-277 \\
-\quad 3\end{array}$ & $\begin{array}{l}-13 \\
+10\end{array}$ & $\begin{array}{l}-40.8 \\
-39.9\end{array}$ & $\begin{array}{l}-3 \\
-76\end{array}$ & $\begin{array}{l}-129 \\
-41\end{array}$ & $\begin{array}{l}-32 \\
+54\end{array}$ \\
\hline $\mathbf{B M}$ & $0-7$ & 48 & 12 & 100 & +8 & +2 & +290 & +15.3 & -0.1 & -3 & -11 & +16.1 & +5 & +301 & +263 \\
\hline JM & $0-7$ & 487 & 12 & 300 & -104 & -218 & -229 & -68.5 & -0.6 & -48 & -13 & -70.6 & -170 & -216 & -48 \\
\hline
\end{tabular}

* Balance of $\mathrm{N}$ in excess of transfers of NPN

Values corrected for anabolism and catabolism of protein

$\ddagger$ Received $\mathrm{H}$ cycle resin instead of $\mathrm{NH}_{4}$

ments observed in the control studies. This same difference was still present in those of our patients who took comparable amounts of the resin while being treated with medications which replace or augment some of the effects of the adrenal cortex (Table VI).

\section{E. Balance data during the ingestion of acidifying and non-acidifying resins without and with hormones or steroids}

The information available on external exchanges is limited by the incompleteness of intake data in the studies in which equal amounts of hydrogen and potassium form resin were given but is nonetheless quite clear on the following four points (Table VII) : $a$ ) with but one exception periods characterized by ingestion of these particular resin mixtures were also characterized by positive balances of potassium; $b$ ) in general, the retained potassium entered the cellular or, more accurately, the extrachloride space; $c$ ) the sodium balances were still positive despite resin intake in the subject receiving significant amounts of these electrolytes (DL, periods 6-9 and 9-11 in Table VII) from dietary sources; and $d$ ) this resin mixture did not accelerate the losses of sodium, chloride, and water already evident in subject DY on essentially complete sodium restriction (Table VII).

The next four experiments listed in Table VIII represent studies in which the smaller amounts of a potassium cycle carboxylic cation exchanger, 12 to $15 \mathrm{~g}$., were added to a daily intake of 45 to $48 \mathrm{~g}$. of the ammonium cycle resin. On this mixture the external balances of potassium were positive in three of the four experiments in which adequate intake data are available, even though the experimental periods were only three to seven days long. Similarly, the balances of both sodium and chloride were negative only in RT, whereas FC retained sodium without chloride.

In the final set of studies in Table IX (patients $\mathrm{BS}, \mathrm{BM}$, and JM), in which intake data are available during combined ACTH or cortisone and resin therapy, the external exchanges of potassium, sodium, and chloride followed the variable patterns observed in the $80: 20$ studies in subjects not given either the hormone or the steroid, but there was a greater incidence of negative potassium and sodium balances, with all subjects losing potassium from their extracellular fluid. It will also be recalled that these patients as a group developed hypokaliemia.

\section{DISCUSSION}

It is readily evident from the above results that we can speak only of trends and implications. First, our data in general indicate that the inclusion of some potassium form resin in a resin mixture will mitigate or cancel the losses of endogenous potassium which result when either the hydrogen or ammonium cycle exchangers are used alone for the purpose of reducing sodium absorption. It is obvious that this effect will be observed only if the potassium present on and in the resin is replaced by another cation and thereby made available for absorption. That this does occur to a variable degree cannot be denied. It seems reasonable to suggest, moreover, that the provision of an ade- 
quate intake of potassium could perhaps be achieved with greater assurance simply by giving potassium salts at appropriate times during resin therapy. In addition, such an alternative would relieve the patient of some of the burden of ingesting the large amounts of resin currently necessary for the removal of clinically significant amounts of sodium (9). Be that as it may, the trend at present seems to be toward admixtures of the potassium charged exchangers with the ammonium or hydrogen form resins (10-12). It behooves us, therefore, to continue to examine our data on this point as critically as possible. Such scrutiny indicates that if the intake of the potassium form of the resin is achieved at the expense of the hydrogen or ammonium form, the efficiency of the mixture in augmenting stool sodium decreases. This is suggested in the comparisons of the $50: 50$ and the $80: 20$ mixtures. Furthermore, it should be noted that as a consequence this is a relatively inefficient way of giving potassium since a large proportion of the ingested ion reappears in the stools. This presumably reflects the greater affinity of the carboxylic exchanger for this ion as compared with sodium (1317), but it may also be a manifestation, in part at least, of the preponderance of potassium in formed stools (18).

The above statements apply as well to the studies conducted in patients undergoing treatment with ACTH or with cortisone. It has been established in a sufficiently extensive series that these last two agents, alone or in combination, do not alter the electrolyte or nitrogen content of formed stools (19). Hence it is not surprising that the administration of resin mixtures to such patients also increases stool sodium and potassium by amounts comparable to those noted in subjects not receiving ACTH or cortisone. The net effects of such resin mixtures have been used in attempts by us, as indicated by the results in Table IX, to cancel or retard the trends to sodium retention and to potassium deficits mediated through the renal effects of the adrenocortical steroids. It seems likely that larger proportions of potassium resin should prove more efficacious.

The serum data need but little comment. The replacement of progressively larger amounts of an acidifying form of the resin with a non-acidifying congener does of course minimize the tendency to acidosis and hypochloremia. The degree to which this proves effective will depend not on the proportion of the two resins but on the total amount of acidifying resin ingested. Hence the end effects do not represent a cancellation of the acidifying effects of certain forms of the resin.

The emphasis on prevention or mitigation of the acidosis by means of resin mixtures has several sequellae, some of which may prove undesirable. Thus we have already discussed the decreased effect on stool sodium. Also, evidence is available that the acidosis produced by the resin facilitates delivery of edema. Finally, if the acidosis and hyperchloremic effects are lost, they cannot be used to counteract (leaving aside for the moment the question of whether or not this serves any useful purpose) the opposite changes which appear in prolonged cortisone or ACTH therapy.

Finally a word of warning is in order, since under some circumstances any procedures which augment body potassium stores are contraindicated. This is particularly true in those patients with renal failure and a tendency toward potassium accumulation (20-22). It may be predicted with assurance that such subjects would be harmed by therapy with potassium cycle exchanger, since toxic levels of this cation would be reached that much sooner. This danger of course would not be present in patients with normal or even an increased ability to excrete potassium (23-25). From the practical point of view, however, it would be well not to use the potassium cycle exchanger in any patient with renal failure until the adequacy of the renal mechanisms for the disposal of excesses of this cation has been established.

\section{SUMMARY AND CONCLUSIONS}

Hydrogen or ammonium form carboxylic cation exchangers have been mixed with the potassium form of the resin in various proportions and administered to patients:

1. The acidosis and hyperchloremia do not appear in short-term therapy with $1: 1$ mixtures in which the total dosage is $60 \mathrm{~g}$. or less, and yet stool sodium is increased to some extent.

2. With 4:1 mixtures in which the acidifying resin predominates, acidosis and hyperchloremia develop and the output of stool sodium rises well above control values. This is also true of stool 
potassium indicating that only part of the potassium form resin has exchanged for cations other than potassium.

3. With the addition of the potassium form resin to hydrogen or ammonium forms, the external balances of potassium are generally positive in all of the mixtures tested.

4. The effects of the resin mixtures on the composition of stools were still evident in patients receiving ACTH or cortisone.

5. The clinical utility of these particular resin mixtures in preventing or deferring the sodium retention, the potassium depletion, the hypochloremia and the alkalosis of prolonged ACTH or cortisone studies has been discussed. The danger of inducing potassium depletion during such therapy, if a sufficiently large intake of potassium is not provided, has been emphasized.

\section{REFERENCES}

1. Greenman, L., Peters, J. H., Mateer, F. M., Weigand, F. A., Wilkins, D., Tarail, R., Rhodes, G., and Danowski, T. S., Biochemical changes accompanying the ingestion of a carboxylic cation exchanger in the hydrogen, ammonium, sodium, potassium, or calcium form. J. Clin. Invest., 1951, 30, 995.

2. Danowski, T. S., Greenman, L., Mateer, F. M., Parsons, W. B., Weigand, F. A., Mermelstein, H., and Peters, J. H., Carboxylic cation exchange resin effects in dogs. J. Clin. Invest., 1951, 30, 984.

3. Danowski, T. S., Greenman, L., Mateer, F. M., Peters, J. H., Weigand, F. A., Mermelstein, H. A., and Clarke, C. E., Carboxylic cation exchange resin studies in animals and humans. J. Clin. Invest., 1950, 29, 807.

4. Danowski, T. S., Cation exchange resins in experimental and clinical situations with special emphasis on edema. Proc. 18th International Physiological Congress, p. 173. Bianco lunos, Copenhagen, 1950.

5. Mote, J. R., Proceedings of the First Clinical ACTH Conference. The Blakiston Company, Philadelphia, Pa., 1950.

6. Danowski, T. S., in discussion on Wilson, May G., and Helper, Helen N., The effect of ACTH in acute carditis. Chapter 49, pp. 640-648. Second Clinical ACTH Conference. The Blakiston Co., Philadelphia, 1951, Vol. 2.

7. Sayers, G., The adrenal cortex and homeostasis. Physiol. Rev., 1950, 30, 241.

8. Greenman, L., Danowski, T. S., Tarail, R., Peters, J. H., Weigand, F. A., and Mateer, F. M., Studies of prevention of some of the toxic effects of pro- longed cortisone and ACTH therapy. J. Clin. Invest., 1951, 30, 644.

9. Danowski, T. S., Greenman, L., Peters, J. H., Mateer, F., Weigand, F. A., and Tarail, R., The use of cation exchange resins in clinical situations. Ann. Int. Med., in press.

10. Chapman, D. W., and Pannill, C. H., The effect of a new cation exchange resin on edematous states and electrolyte balance. J. Lab. \& Clin. Med., 1950, 36, 808.

11. Martz, B. L., Kohlstaedt, K. G., and Helmer, O. M., Use of ion exchange resins in the management of congestive heart failure and cirrhosis of the liver. J. Lab. \& Clin. Med., 1950, 36, 962.

12. Hay, S. H., and Wood, J. E., Jr., Cation exchange resins in the treatment of congestive heart failure. Ann. Int. Med., 1950, 33, 1139.

13. Bauman, W. C., and Eichhorn, J., Fundamental properties of a synthetic cation exchange resin. J. Am. Chem. Soc., 1947, 69, 2830.

14. Kunin, R., Ion exchange. Anal. Chem., 1949, $21,87$.

15. Boyd, G. E., Schubert, J., and Adamson, A. W., The exchange adsorption of ions from aqueous solutions by organic zeolites. I. Ion-exchange equilibria. J. Am. Chem. Soc., 1947, 69, 2818.

16. Nachod, F. C., Ion Exchange: Theory and Application. Academic Press, Inc., New York, 1949.

17. Kunin, R., and Myers, R. J., Ion Exchange Resins. John Wiley \& Sons, Inc., New York, 1950.

18. Danowski, T. S., and Elkinton, J. R., Exchanges of potassium related to organs and systems. Pharmacol. Rev., 1951, 3, 42.

19. Danowski, T. S., and Greenman, L., Unpublished data.

20. Finch, C. A., Sawyer, C. G., and Flynn, J. M., Clinical syndrome of potassium intoxication. Am. J. Med., 1946, 1, 337.

21. Elkinton, J. R., Tarail, R., and Peters, J. P., Transfers of potassium in renal insufficiency. J. Clin. Invest., 1949, 28, 378.

22. Keith, N. M., and Burchell, H. B., Clinical intoxication with potassium: its occurrence in severe renal insufficiency. Am. J. M. Sc., 1949, 217, 1.

23. Brown, M. R., Currens, J. H., and Marchand, J. F., Muscular paralysis and electrocardiographic abnormalities resulting from potassium loss in chronic nephritis. J. A. M. A., 1944, 124, 545.

24. Albright, F., Burnett, C. H., Parson, W., Reifenstein, E. C., Jr., and Roos, A., Osteomalacia and late rickets. The various etiologies met in the United States with emphasis on that resulting from a specific form of renal acidosis, the therapeutic indications for each etiological sub-group, and the relationship between osteomalacia and Milkman's syndrome. Medicine, 1946, 25, 399.

25. Leaf, A., and Camara, A. A., Renal tubular secretion of potassium in man. J. Clin. Invest., 1949, 28, 1526. 